\title{
Diseño de una celda electrolítica para aplicación de recubrimientos metálicos
}

\author{
Adonai Zapata Gordon; José Luis Tristancho Reyes²*; Facundo Almeraya Calderón² \\ ${ }^{1}$ Facultad de Ingeniería Mecánica, Universidad Tecnológica de Pereira (UTP), Cra. 27 \#10-02, Pereira, Colombia \\ ${ }^{2}$ Facultad de Ingeniería Mecánica y Eléctrica, Universidad Autónoma de Nuevo León, Avenida Universidad s/n, Cd. \\ Universitaria, San Nicolás de los Garza, Nuevo León C.P. 66451, Monterrey, México. \\ *josetrs@utp.edu.co
}

Fecha recepción: mayo 28 de 2019 Fecha aceptación: mayo 15 de 2020

\section{Resumen}

En esta investigación se realizó el diseño de una nueva configuración de celda de laboratorio para la aplicación de recubrimientos electrolíticos metálicos tales como: electrozincado, cromado, cobrizado, anodizado, entre otros. Para el diseño de esta celda se delimitaron variables con rangos específicos como: un volumen entre 100 y $500 \mathrm{ml}$ de solución electrolítica, un tamaño de cátodo entre 0,1 y 0,33 $\mathrm{dm}^{2}$ de área expuesta, ánodos entre 1 y 2 veces el área del cátodo, se adecuó a la celda una fuente de corriente con densidad máxima de $9 \mathrm{~A} / \mathrm{dm}^{2}$, además, presenta una variación en la distancia entre ánodo y cátodo mediante guías deslizantes para optimización de los procesos de investigación. El recipiente donde se deposita la solución electrolítica, en celda está fabricado en refractario tipo Pyrex y la cubierta de la misma fue fabricada en ABS (Acrylonitrile Butadiene Styrene) para evitar su deterioro por efectos de la corrosión debida a las soluciones electrolíticas, para el posicionamiento de los electrodos se diseñó un soporte porta electrodos (porta ánodo y porta cátodo) con características intercambiables para múltiples ánodos y cátodos según lo exija la experimentación. Para validar el adecuado comportamiento de la celda se realizó la deposición de un recubrimiento zinc sobre acero 1020 obteniendo un electrozincado homogéneo y continuo. La celda de electrorecubrimiento permite experimentar diferentes tipos de sustratos como aceros, aleaciones de aluminio ó titanio, entre otros, en aplicaciones industriales finales o en preprocesos.

Palabras clave: Celda Electrolítica; Electrorecubrimiento; Diseño. 


\title{
Design of an electrolytic cell for the application of metal coatings
}

\begin{abstract}
In this research, the design of a new laboratory cell configuration for the application of metallic electrolytic coatings such as: electroplating, chrome plating, copper plating, anodizing, among others. For the design of this cell, variables with specific ranges such as: a volume between 100 and $500 \mathrm{ml}$ of electrolytic solution, a cathode size between 0.1 and $0.33 \mathrm{dm}^{2}$ of exposed area, anodes between 1 and 2 times the cathode area, were defined. A current source with a maximum density of $9 \mathrm{~A} / \mathrm{dm}^{2}$. Was adapted to the cell, in addition, it presents a variation in the distance between anode and cathode by means of sliding guides to optimize the investigation processes. The container where the electrolytic solution is deposited, in the cell is made of Pyrex type refractory and its cover was made of ABS (Acrylonitrile Butadiene Styrene) to avoid deterioration due to the effects of corrosion due to electrolytic solutions. For the positioning of the electrodes, it was designed an electrode holder (anode holder and cathode holder) with interchangeable characteristics for multiple anodes and cathodes as required by experimentation. To validate the proper behavior of the cell, a Zinc coating on 1020 steel was deposited, obtaining a homogeneous and continuous electrozinc coating. The electrocoating cell allows to experiment with different types of substrates such as steels, aluminium or titanium alloys, among others, in final industrial applications or in pre-processes.
\end{abstract}

Keywords: Electrolytic Cell; Electrocoating; Design.

\section{Projeto de uma célula eletrolítica para aplicação de revestimentos metálicos}

\begin{abstract}
Resumo
Nesta pesquisa, foi realizado o projeto de uma nova configuração de células laboratoriais para aplicação de revestimentos eletrolíticos metálicos, tais como: galvanoplastia, cromagem, cromagem, anodização, entre outros. Para o projeto desta célula, foram definidas variáveis com faixas específicas, tais como: volume entre 100 e $500 \mathrm{ml}$ de solução eletrolítica, tamanho do cátodo entre 0,1 e 0,33 dm² de área exposta, ânodos entre 1 e 2 vezes a área do cátodo. Uma fonte de corrente com densidade máxima de $9 \mathrm{~A} / \mathrm{dm}^{2}$ foi adaptada à célula, além disso, apresenta uma variação na distância entre o ânodo e o cátodo por meio de guias deslizantes para otimizar os processos de investigação. O recipiente onde a solução eletrolítica é depositada, na célula, é refratário ao tipo Pyrex e sua cobertura é feita em ABS (Acrylonitrile Butadiene Styrene) para evitar deterioração devido aos efeitos da corrosão por soluções eletrolíticas.Para o posicionamento dos eletrodos, foi projetado um suporte de eletrodo (suporte de ânodo e suporte de cátodo) com características intercambiáveis para vários ânodos e cátodos, conforme exigido pela experimentação. Para validar o comportamento adequado da célula, foi depositado um revestimento de zinco no aço 1020, obtendo um revestimento eletro-zinco homogêneo e contínuo. A célula de eletrocoating permite experimentar diferentes tipos de substratos, como aços, ligas de alumínio ou titânio, entre outros, em aplicações industriais finais ou em pré-processos.
\end{abstract}

Palavras-chave: Célula Eletrolitica; Eletrocoating; Design. 


\section{Introducción}

La corrosión es un factor crítico en la fabricación de componentes de la industria automotriz, aeronáutica, farmacéutica y de alimentos. Este deterioro es causado principalmente por las condiciones ambientales y las características propias del material, donde, de forma natural, el material pierde sus propiedades mecánicas, dando lugar a fallas y por consiguiente accidentes muchas veces inesperados.

La industria en Colombia avanza en la fabricación de componentes automotrices y aeronáuticos [8,9], con materiales que sufren desgaste por corrosión; esta es la causa y necesidad de mejorar las características superficiales de estos materiales. El objetivo de este estudio es mejorar, proteger y alargar la vida útil de los componentes que hacen parte de aeronaves y vehículos, a través de la aplicación de recubrimientos metálicos, los cuales pueden incrementar significativamente su tiempo de vida útil. Para el logro del objetivo planteado anteriormente se diseñó y construyó una celda electrolítica para recubrir componentes con alto contenido de hierro debido a que este es un proceso económico comparado con otros procesos de aplicación de recubrimientos como, por ejemplo, la proyección térmica; además de proteger los materiales logra reducir costos de mantenimiento que representan alrededor del 30\% anual del valor de una aeronave o de un vehículo. El recubrimiento, mediante procesos electrolíticos, es un método para proteger materiales u objetos con una fina película de otro material (metal); el principio de este proceso es la electrolisis, conformada por cinco (5) elementos principales: un baño electrolítico, un ánodo ( $\mathrm{Zn}, \mathrm{Al}$, etc), un cátodo (aceros, aleaciones de aluminio, etc) y una fuente de energía eléctrica (corriente y potencial), además de la celda para electrolisis. El objetivo del proceso es lograr una fina y homogénea capa de partículas desprendidas desde el ánodo hacia el cátodo controlando variables como: potencial, corriente, distancia, tiempo, $\mathrm{pH}$, agitación del baño, eficiencia del baño y finalmente la morfología y espesor deseadas según la aplicación [1,2].

\section{Metodología Experimental}

\section{Selección de materiales}

Como criterio para la selección de los materiales se establecieron varios aspectos:

- Resistente a la corrosión

- Resistente a los ácidos

- Resistente a las sales

- Resistente a las bases

- Fácil de replicar

Tabla 1. Selección de material de la cuba o recipiente.

\begin{tabular}{llll}
\hline & Polimetilmetacrilato & Vidrio Pyrex & Polietileno de alta densidad \\
\hline Ácidos & Baja & Alta & Baja \\
Bases & Baja & Alta & Muy baja \\
Sales & Alta & Alta & Buena \\
Corrosión & Alta & Muy alta & Muy Alta \\
Aislante & Alta & Alta & Bueno \\
Manchado & Baja & Muy alta & Alta \\
\hline
\end{tabular}

Fuente: Los Autores

Para el recipiente contenedor de los electrolitos (cuba) se seleccionó el vidrio refractario tipo Pyrex según tabla 1; además, se utilizó el ABS (Acrylonitrile Butadiene Styrene) para la cubierta con el objeto de poder imprimir el modelo con tecnologías modernas de impresión en 3D, según los requerimientos de diseño [3].

\section{Diseño de prototipo}

Selección área catódica. Para delimitar la investigación se estableció el área a recubrir en el cátodo mediante la ecuación 1 (figura 1) [4].

$$
\begin{gathered}
L=37 \mathrm{~mm}, H=55 \mathrm{~mm}, H_{R}=45 \mathrm{~mm} \\
\text { Area }_{R}=L * H_{R} \\
\text { Area }_{R}=0,37 \mathrm{dm} \times 0,45 \mathrm{dm}=0,1665 \mathrm{dm}^{2}
\end{gathered}
$$




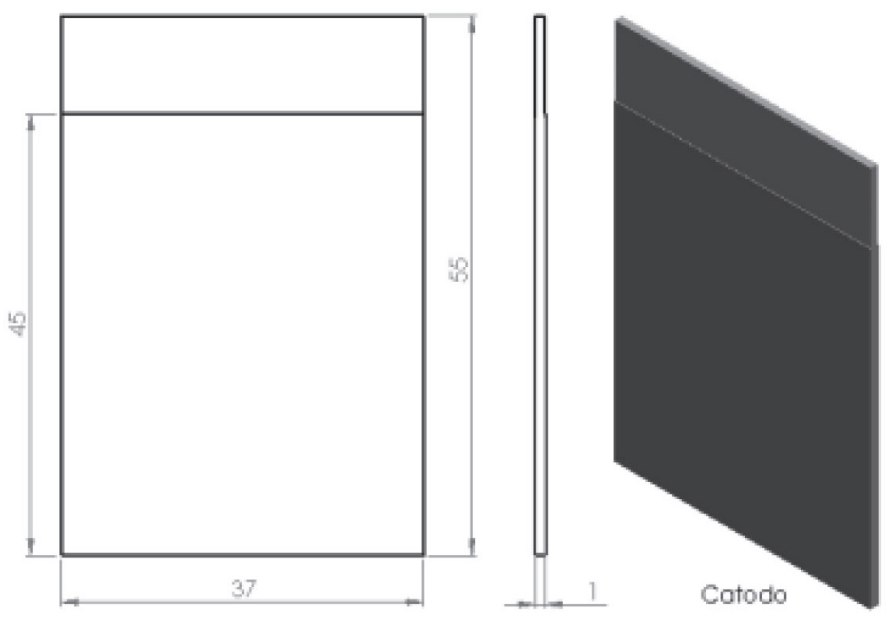

Figura 1. Dimensiones del cátodo o sustrato.

Se determinó el área del cátodo, en base a las posibles pruebas que se pueden extraer de esta como: ASTM E797 / E797M-15 [10], ASTM F1375-92 [11], ASTM B117-11 [12], ASTM D3359-09e2 [13], ASTM G5-94 [14], ASTM G59 - 97 [15], ASTM G102 - 89 [16], 2015, ASTM G1 - 03 [17].

Diseño cuba. La reproducibilidad es un factor fundamental en la industria que permite dar garantía de las características de un proceso o un producto; debido a ello se diseñó una cuba, figura 2, para contener los electrolitos y poder llevar acabo la aplicación de los recubrimientos electrolíticos tomando como base el cálculo y tamaño de espécimen a recubrir.

Diseño de la cubierta. Con base en la cuba se realizó un diseño de la cubierta teniendo en cuenta varios aspectos:

- Sistema porta electrodo (porta ánodos y porta cátodo).

- Sistema de tapa soporte.

Los diseños de los porta electrodos (porta ánodos y porta cátodo) se realizaron con base en el tamaño y forma del sustrato seleccionado con el objeto de hacer control del área anódica (figura 3).
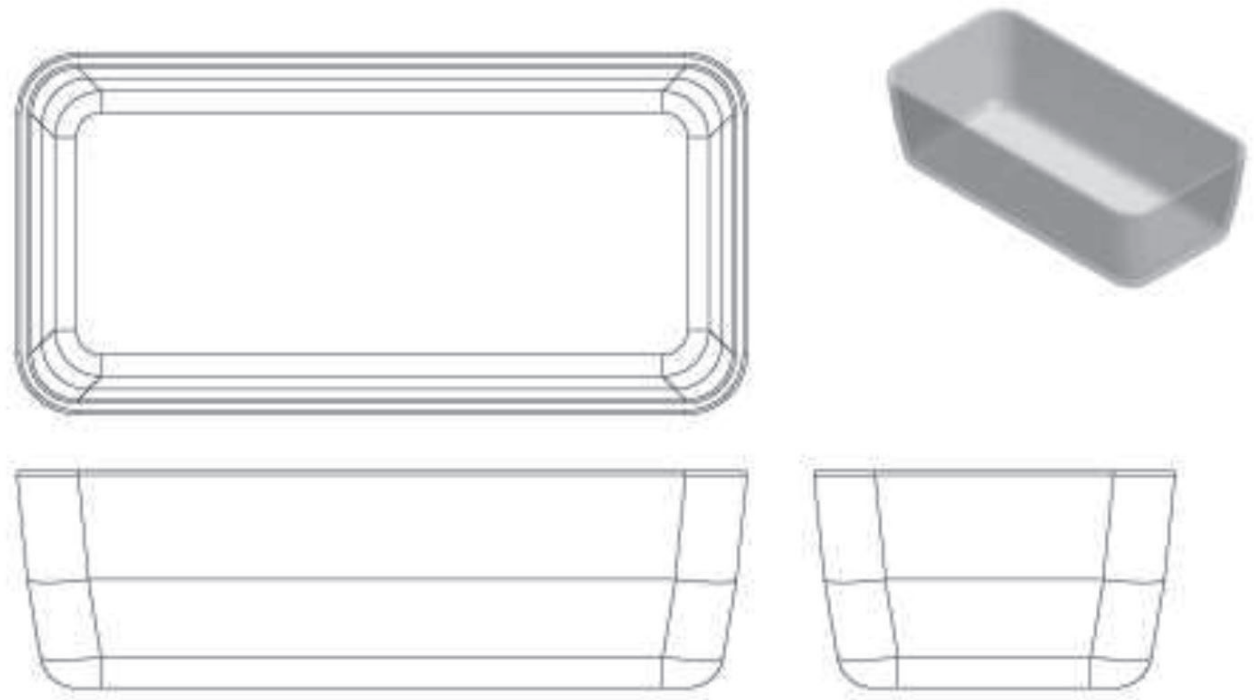

Figura 2. Diseño de la cuba. 


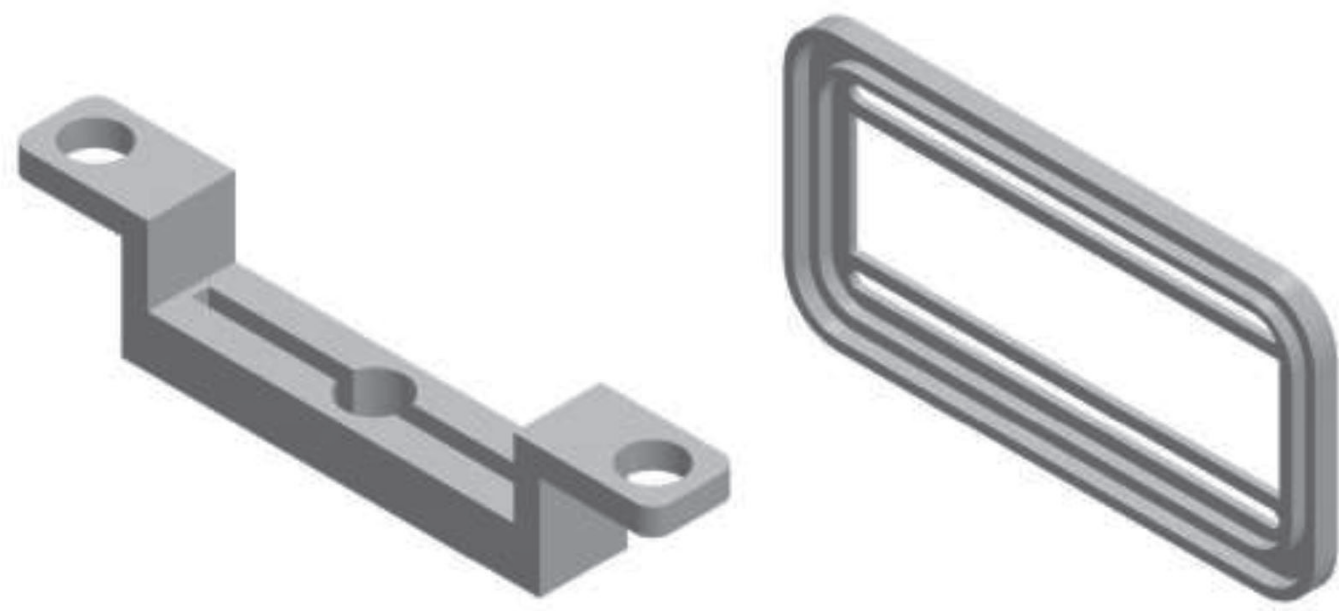

Figura 3. Diseño soportes porta electrodos (porta ánodos y porta cátodo) y tapa de soporte.

Para el diseño de la tapa soporte, figura 3, se hizo con base a los porta electrodos (porta ánodos y porta cátodo), además, de permitir ajustar la distancia entre ánodo y cátodo, dando así mayor versatilidad en las posibles experimentaciones. En la figura 4 se puede observar el proceso de obtención de los porta electrodos (porta ánodo y cátodo) en el material ABS, mediante la tecnología de modelado en un proceso de impresión 3D.

El conjunto de la cuba, la tapa soporte y los porta electrodos (porta ánodos y porta cátodo) se muestran en la figura 4, estos últimos posicionados de tal forma que el sustrato (material a recubrir) este en el centro de la celda y los ánodos a los extremos de la celda.

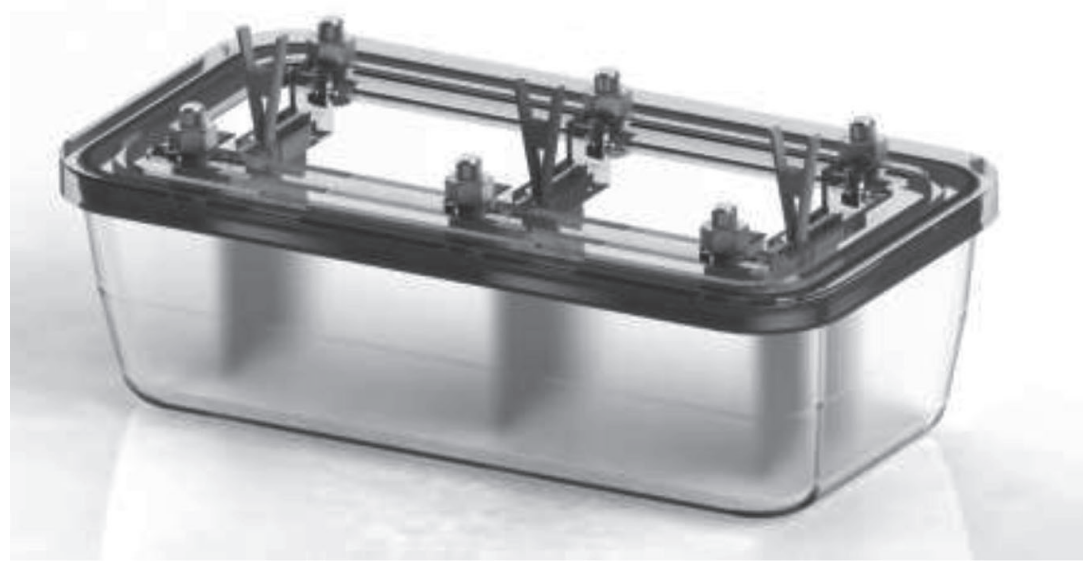

Figura 4. Diseño celda para electrorecubrimientos.

\section{Resultados y discusión}

\section{Construcción celda electrolítica}

Los resultados obtenidos durante la construcción de las partes de la celda para electrorecubrimientos se muestran en las figuras 5 y 6 , donde se observa la impresión en ABS a través de las tecnologías de impresión 3D. La figura 7 muestra el ensamble completo de la celda diseñada y construida para la aplicación de recubrimientos electrolíticos. 


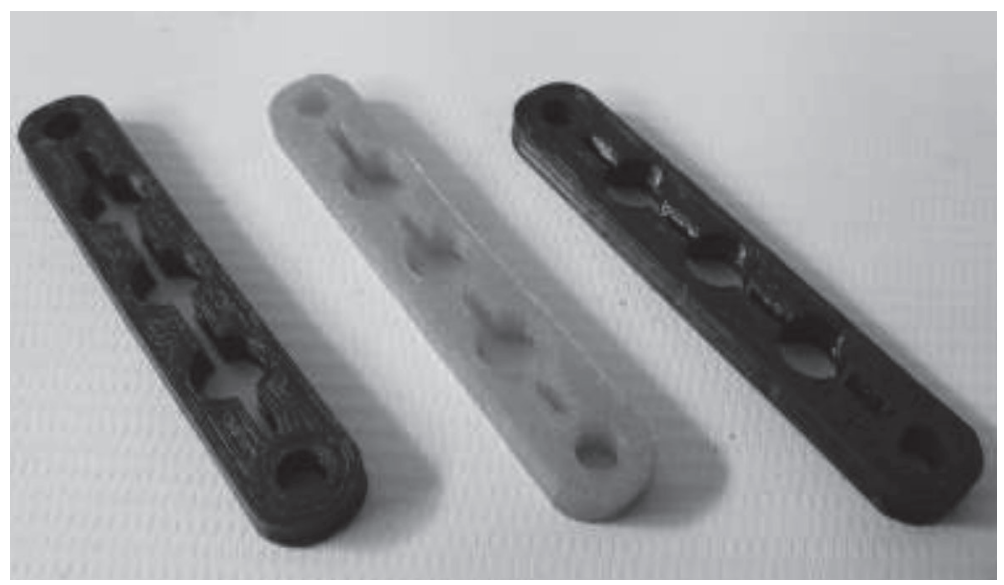

Figura 5. Porta electrodos (porta ánodos y porta cátodo).

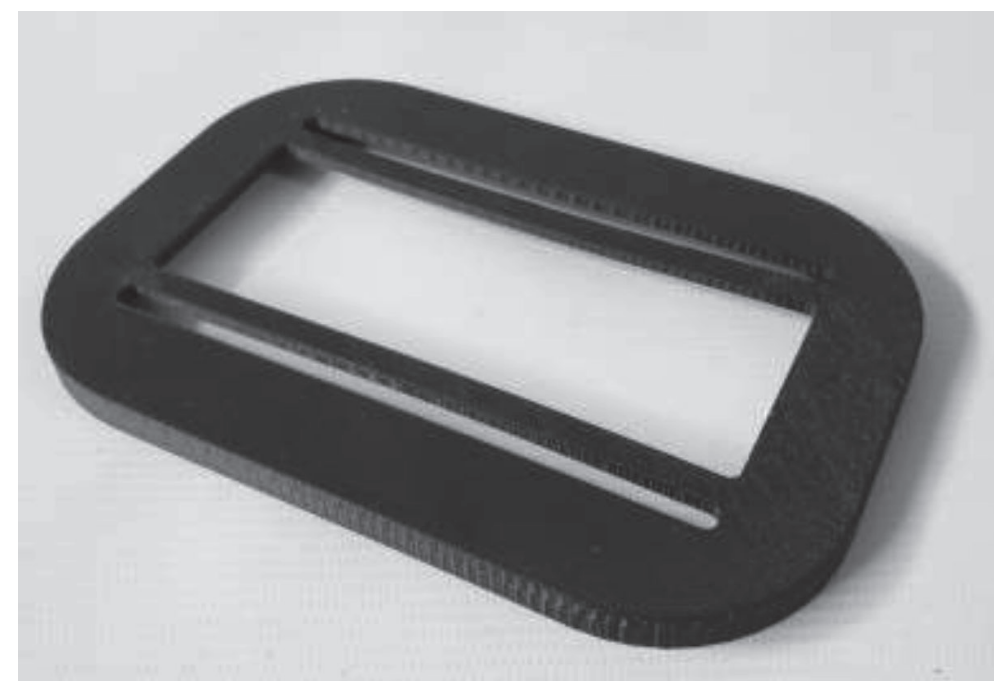

Figura 6. Tapa Soporte.

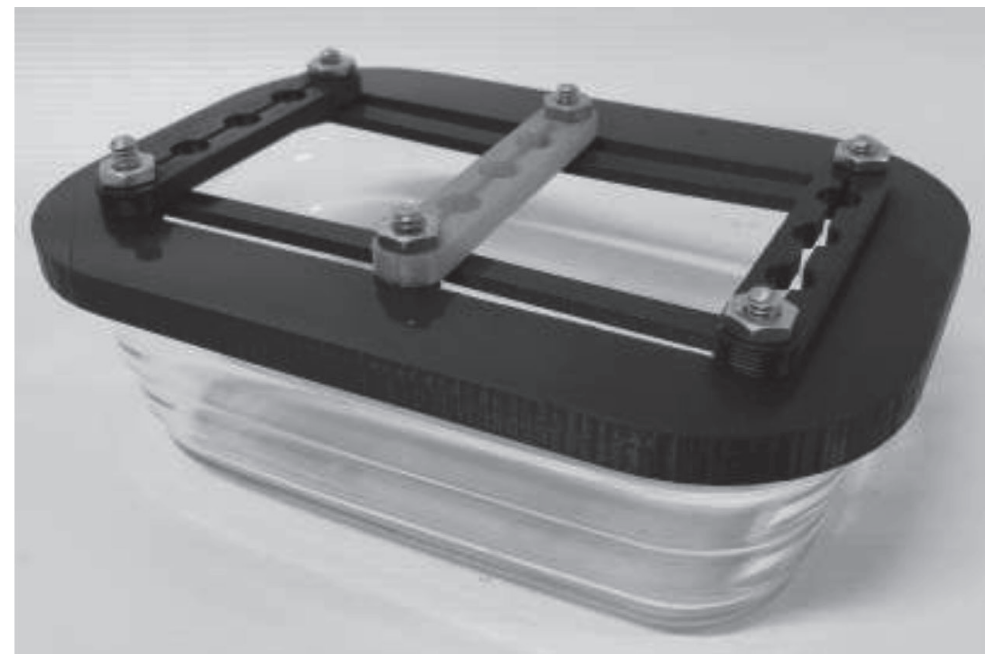

Figura 7. Celda para recubrimientos electrolíticos. 
Pruebas de funcionamiento. Para validar el diseño se realizaron varias pruebas de funcionamiento involucrando como material base (sustrato) acero AISI-SAE 1020 y dos electrodos anódicos de zinc al 99\%, figura 8.

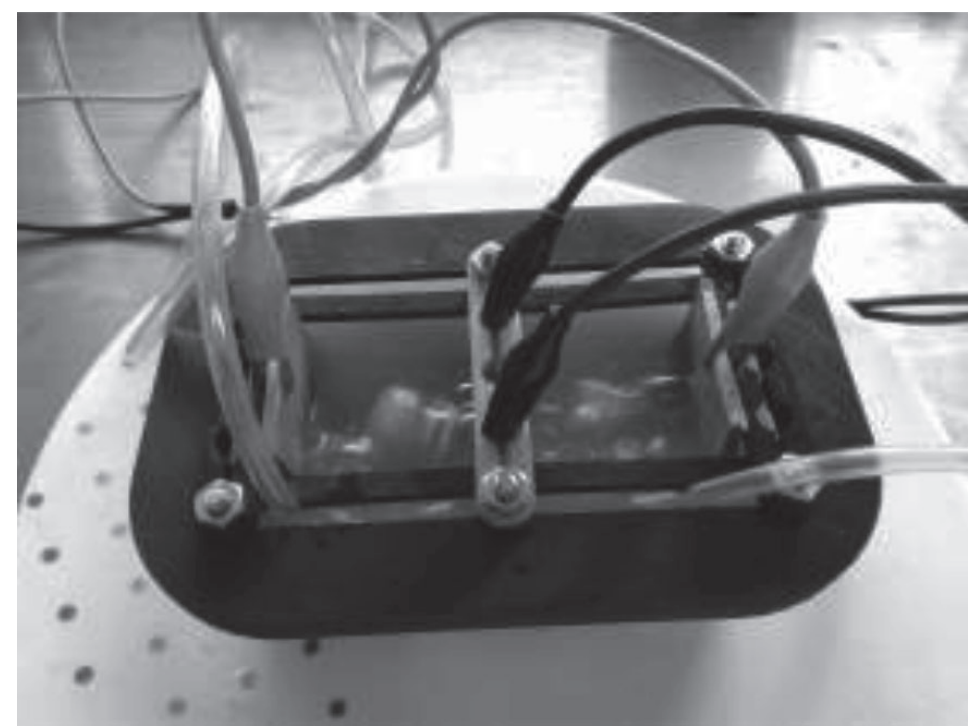

Figura 8. Montaje de prueba de funcionamiento de la celda.

Una de las probetas antes de ser sometida al proceso de deposición de zinc electrolítico y después de someterse a este es mostrada en la Figura 9. Se hace evidente la presencia de un recubrimiento homogéneo haciendo variaciones de distancia entre el ánodo y el cátodo, obteniendo espesores experimentales aceptables entre 3 y 8 micrómetros para las probetas con un tiempo de 5 y 10 minutos respectivamente y con la densidad de corriente de $5 \mathrm{~A} / \mathrm{dm}^{2}$.
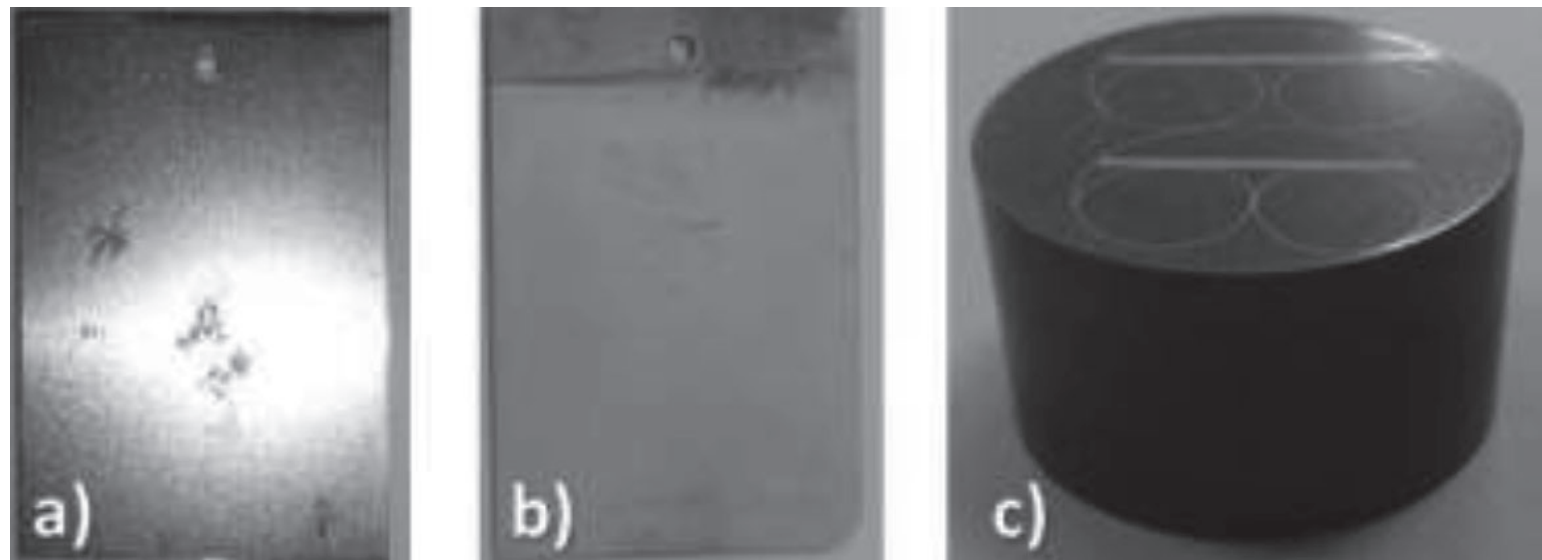

Figura 9. Probeta sin recubrimiento a), con recubrimiento b) y en baquelita c).

\section{Caracterización de recubrimientos electrozincados}

Medición de espesor por ultrasonido. Para lograr determinar el espesor de los recubrimientos obtenidos se hizo uso de un equipo de ultrasonido Elcometer con transductor acoplado (figura 10).
La medición de espesores se realizó en 9 puntos diferentes de la muestra y se obtuvo el promedio de estas mediciones. Los resultados obtenidos en algunos de los recubrimientos depositados se pueden ver en la tabla 2. 
Tabla 2. Valores de medición de espesor.

\begin{tabular}{cc}
\hline Muestra. & Espesor $(\mu \mathrm{m})$ \\
\hline A1 & 5,37 \\
A2 & 7,3 \\
A3 & 5,44 \\
A4 & 9,9 \\
A5 & 8,04 \\
A6 & 6,43 \\
A7 & 8,56 \\
A8 & 7,8 \\
\hline
\end{tabular}

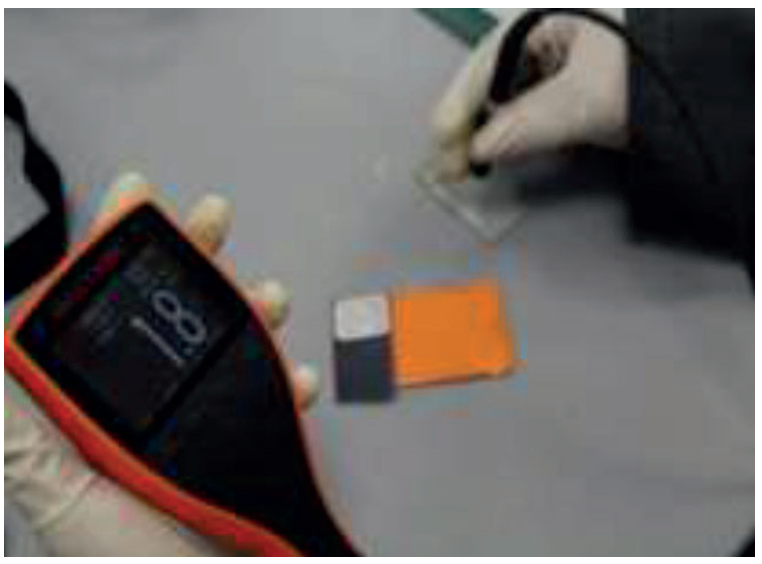

Figura 10. Equipo para medición de espesores Elcometer.

Microscopia electrónica de barrido (MEB). Una vez obtenidas las piezas electrozincadas se prepararon muestras para analizar la morfología superficial y transversal mediante observación en microscopio electrónico de barrido JEOL JSM 5800 .

En la figura 11 se presentan las imágenes superficiales de los recubrimientos electrozincados obtenidos a tiempos de 1,3 y 5 minutos de deposición y con densidad de corriente de $9 \mathrm{~A} / \mathrm{dm}^{2}$. Se observa que el tamaño de grano aumenta conforme se incrementa el tiempo de deposición; los granos son homogéneos en los tres tiempos utilizados, para los tiempos de 3 y 5 min se observan granos hexagonales de tamaños diversos y se presentó crecimiento aleatorio en todos los tiempos de electrodeposición. Para los tiempos de 3 y 5 min se observan claramente los límites de los granos, observación que no ocurre para el tiempo de $1 \mathrm{~min}$. Los análisis EDS de las superficies para las muestras correspondientes a
3 y 5 min muestran zinc en su totalidad, mientras que la muestra de 1 min muestra presenta hierro.

Prueba de corrosión acelerada en cámara de niebla salina. Para la realización de los ensayos acelerados de corrosión en cámara de niebla salina se utilizó un equipo Q-FOG/Q-LAB Cyclic Corrosion Tester.

En la figura 12, se muestran las imágenes transversales de los recubrimientos electrozincados realizados a tiempos de 1,3 y 5 min con densidad de corriente de $9 \mathrm{~A} / \mathrm{dm}^{2}$. Se observa que todos los recubrimientos depositados bajo estas condiciones presentaron agrietamientos que llegaban hasta el sustrato metálico. Se aprecia también un incremento del espesor del recubrimiento en función del tiempo de deposición. Los análisis EDS de la sección transversal muestran la presencia de $\mathrm{Zn}, \mathrm{Cl}, \mathrm{S}$ y $\mathrm{O}$. Adicionalmente se notó la presencia de Si y Al probablemente provenientes de los aditivos utilizados durante la preparación metalográfica. 

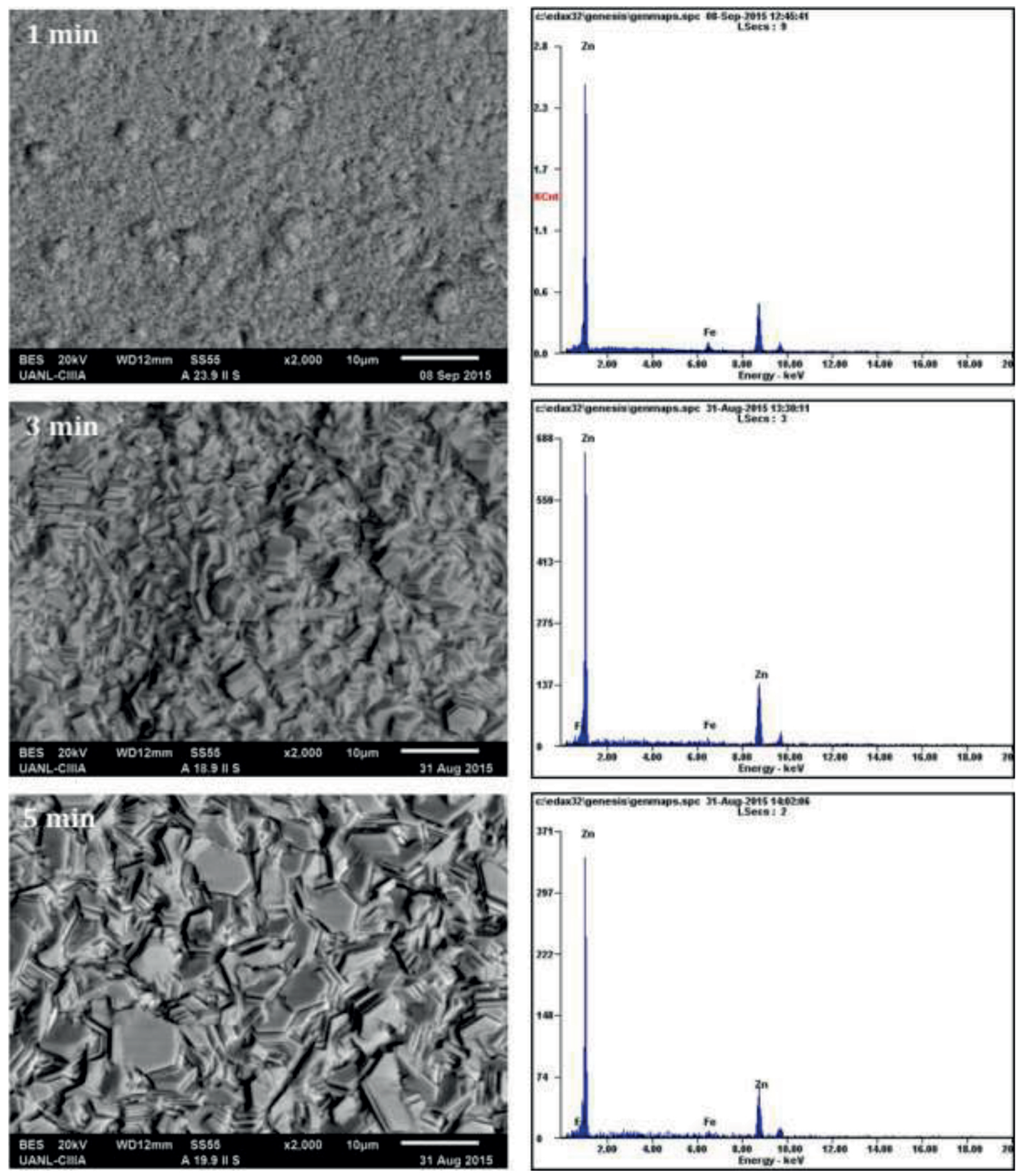

Figura 11. Morfología superficial a 2000X de placa electrozincada con densidad de corriente de $9 \mathrm{~A} / \mathrm{dm}^{2}$ y tiempos de electrodeposición de 1,3 y 5 minutos. 

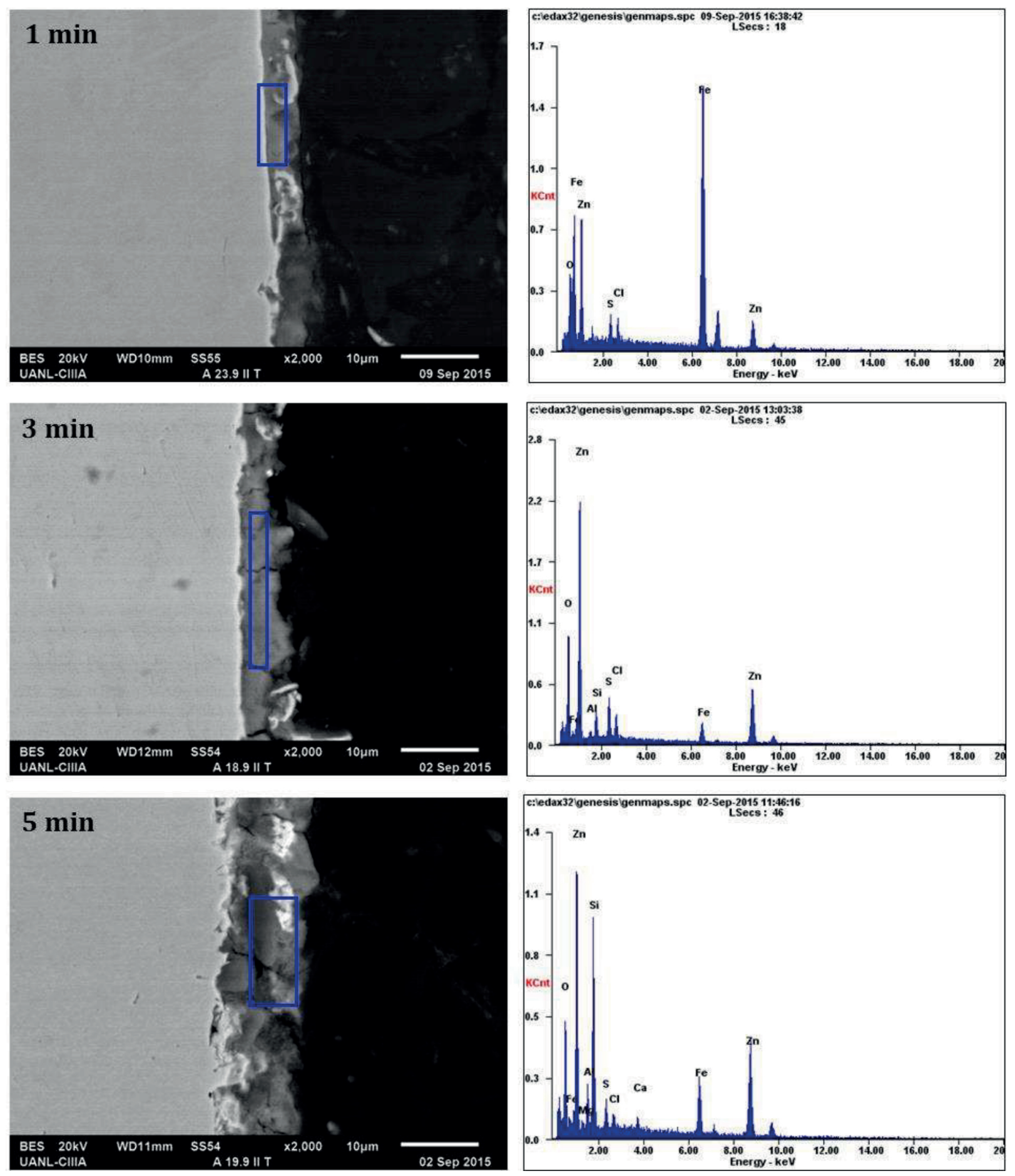

Figura 12. Morfología transversal a 2000X de placa electrozincada con densidad de corriente de $9 \mathrm{~A} / \mathrm{dm}^{2}$ y tiempos de electrodeposición de 1,3 y 5 minutos.

La tabla 3 muestra los resultados obtenidos al someter los recubrimientos obtenidos a tiempos 1,3 y 5 minutos de diferentes muestras electrozincadas. 
Tabla 3. Análisis de resultados obtenidos en cámara de niebla salina.

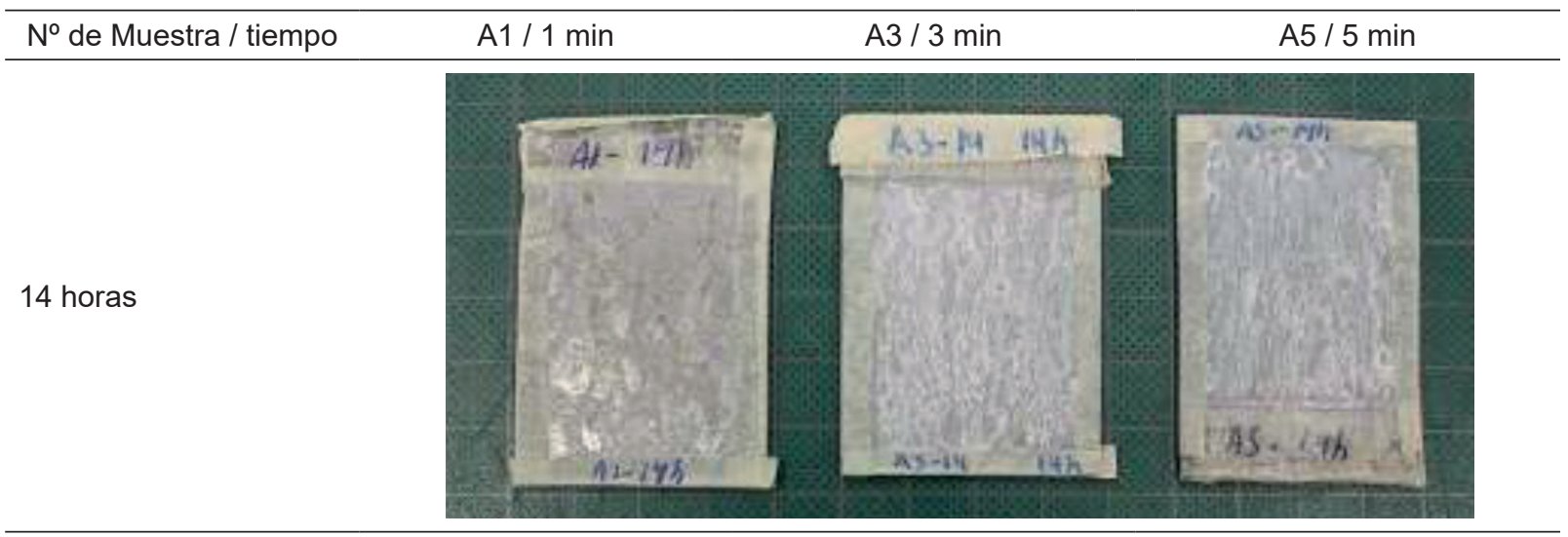

Observación:

Para una exposición de 14 horas se observa la formación inicial de pequeñas zonas de óxido rojo solo en la muestra de $1 \mathrm{~min}$.

16 horas

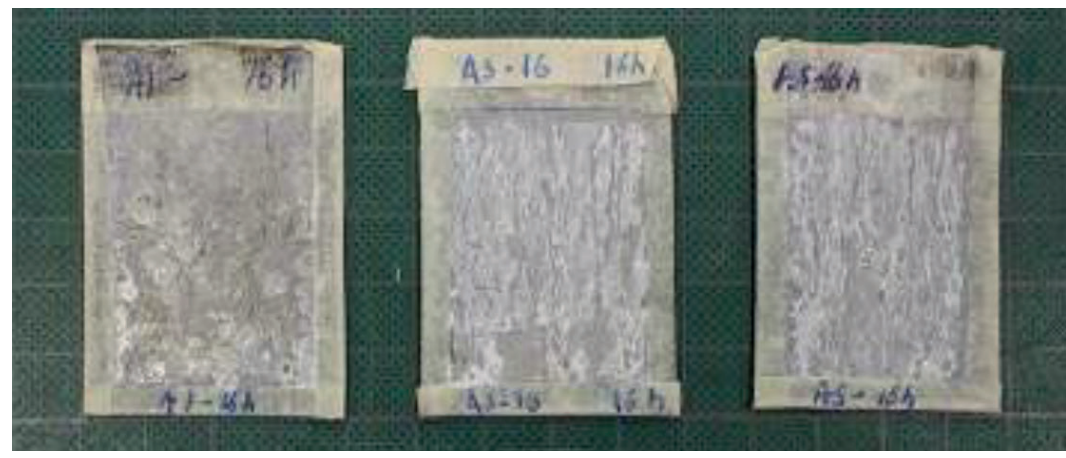

Observación:

Para una exposición de 16 horas se puede ver el óxido rojo formado en la muestra de 1 min y óxido blanco en las demás.

Prueba de adherencia de recubrimiento. La prueba de adherencia de recubrimiento se realizó bajo norma ASTM-D3359.08, correspondiente a una prueba de adherencia por cinta, usando un proceso de rayado de las probetas sometidas a ensayo.
La tabla 4 muestra el análisis de los resultados obtenidos de la prueba de adherencia por cinta de las probetas después de ser sometidas a ensayo de corrosión acelerada en cámara de niebla salina.

Tabla 4. Análisis de resultados obtenidos de la prueba de adherencia por cinta.

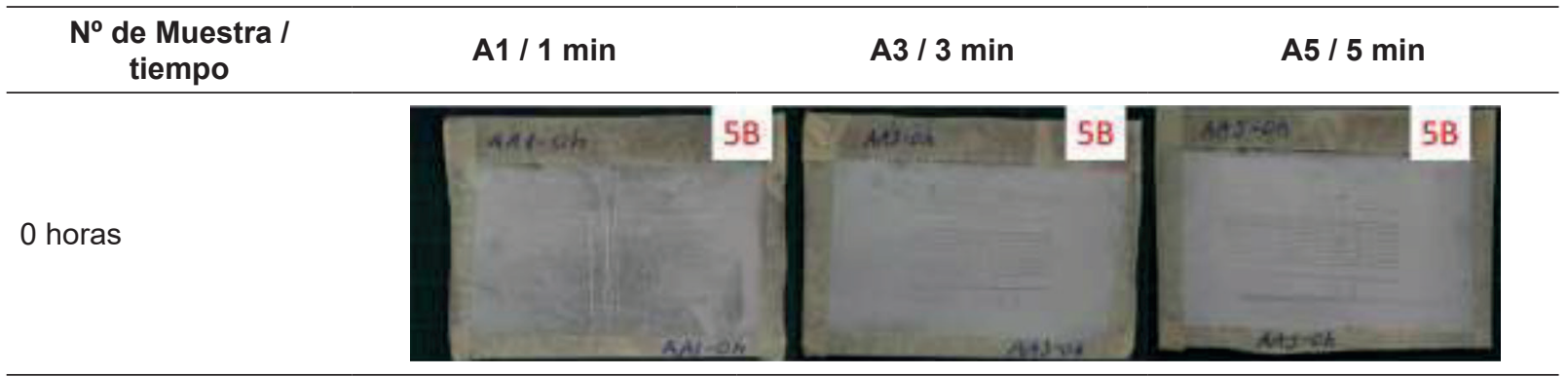

Observación:

En la exposición de 0 horas no se presenta desprendimiento del recubrimiento 


\section{Continuación Tabla 4.}

\begin{tabular}{|c|c|c|c|}
\hline $\begin{array}{c}N^{\circ} \text { de Muestra / } \\
\text { tiempo }\end{array}$ & $\mathrm{A} 1 / 1 \mathrm{~min}$ & A3 / $3 \mathrm{~min}$ & A5 / $5 \mathrm{~min}$ \\
\hline 4 horas & & & \\
\hline
\end{tabular}

Observación:

Después de 4 horas no presenta desprendimiento del recubrimiento, pero se observa la presencia de óxidos de zinc.

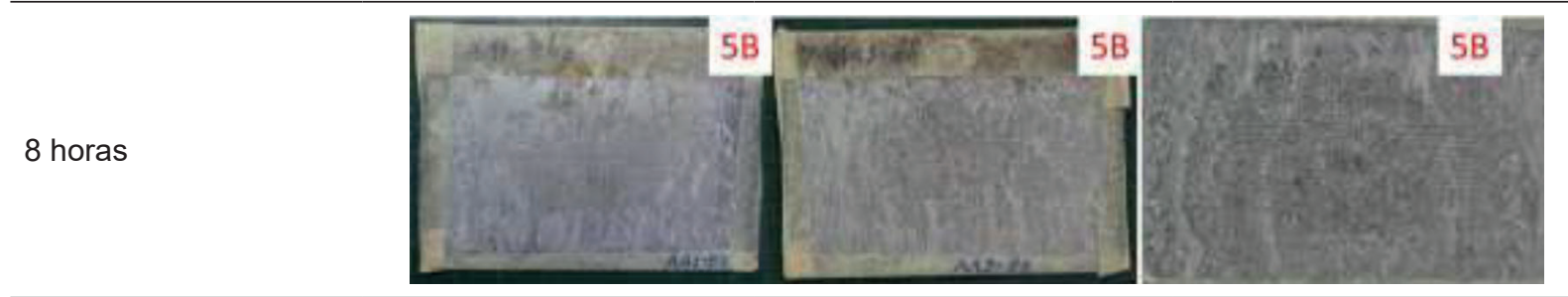

Observación:

Después de 8 horas no se evidencia desprendimiento, pero se observa la presencia óxido blanco en todas las muestras

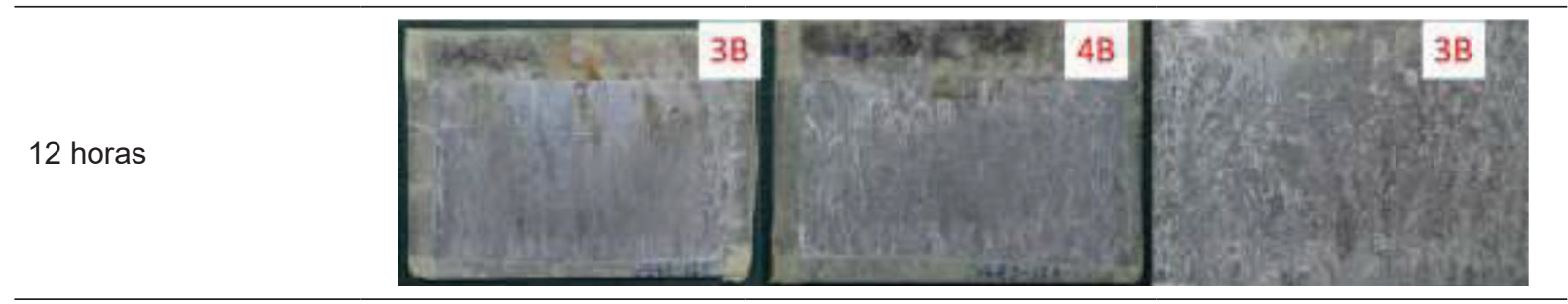

Observación:

En la exposición de 12, horas no hay desprendimiento, pero se observa la coloración del óxido rojo en B1.

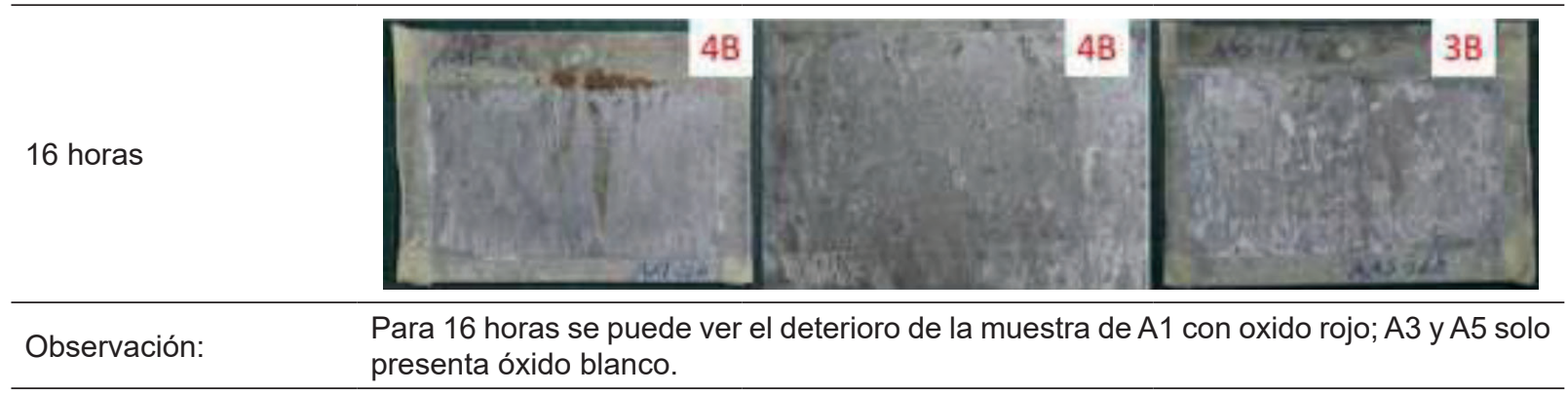

\section{Conclusiones}

La corrosión es un proceso natural de muchos materiales, que tienden a regresar a su estado inicial (óxidos metálicos), por lo que se requiere mejorar $u$ optimizar las tecnologías de la industria dedicada a los recubrimientos, en específico los electrolíticos controlando variables como: la solución, el tiempo, la tensión, la corriente, el $\mathrm{pH}$ y la temperatura a través de proyectos de investigación que involucren nuevos desarrollos y pruebas de laboratorio.

El diseño de la celda permite realizar pruebas a nivel laboratorio, donde a pequeña escala se pueden hacer múltiples variaciones y combinaciones de los procesos de recubrimiento electrolítico, es decir combinar tiempos con corrientes y diferentes soluciones con electrodos de materiales diversos, pudiéndose en su momento replicar a escala industrial. 
Las pruebas realizadas con zinc electrolítico como recubrimiento para la protección contra la corrosión de acero al carbono AISI SAE 1020 validó el propósito de la celda al obtenerse recubrimientos con espesores aceptables, de buena adherencia y que presentaron buen desempeño al ser sometidos a ensayo acelerado de corrosión en cámara de niebla salina.

\section{Agradecimientos}

Los autores expresan sus agradecimientos al Centro de Investigación e Innovación en Ingeniería Aeronáutica (CIIIA) de la Facultad de Ingeniería Mecánica - Eléctrica de la Universidad Autónoma de Nuevo León UANL y al Grupo de Investigación en Materiales Avanzados (GIMAV - UTP) de la Facultad de Ingeniería Mecánica de la Universidad Tecnológica de Pereira UTP por su aporte en el desarrollo de la presente investigación y su apoyo en la formación de las nuevas generaciones de investigadores.

\section{Referencias bibliográficas}

[1] Muralidhara HB, Naik YA. Studies on nanocrystalline zinc coating. Bull. Mater. Sci. 2008;31(4):585-591.

[2] Muralidhara HB, Balasubramanyam J, Naik YA, Kumar KY, Hanumanthappa H, Veena MS. Electrodeposition of Nanocrystalline Zinc on Steel Substrate from Acid Sulphate Bath and its Corrosion Study. J. Chem. Pharm. Res. 2011;3:433-449.

[3] Molera Solá P. Recubrimiento de los Metales. España: Productica Marcombo S.A; 1990.

[4] Jose A. Ortega M. Corrosión Industrial. España: Productica Marcombo S.A; 1989.

[5] Zhang Z, Leng WH, Cai QY, Cao FH, Zhang JQ. Study of the zinc electroplating process using electrochemical noise technique. J. Electroanal. Chem. 2005;578(2):357-367.

[6] Vasilakopoulos D, Bouroushian M, Spyrellis N. Texture and morphology of pulse plated zinc electrodeposits. J Mater Sci. 2006;41:28692875.

[7] Praveen BM, Venkatesha TV. Generation and Corrosion Behavior of Zn-Nano Sized Carbon Black Composite Coating. Int. J. Electrochem. Sci. 2009;4:258-266.

[8] American Galvanizers Association. Zinc Coatings. Colorado, United States; 2011.

[9] Federal Aviation Administration. Aircraft Cleaning y Corrosion Control - Corrosion control. United States: Federal Aviation Administration; 2018.

[10] ASTM E797 / E797M-15. Standard Practice for Measuring Thickness by Manual Ultrasonic Pulse-Echo Contact Method. United States: ASTM International; 2015.

[11] ASTM F1375-92. Método de prueba estándar para la dispersión de energía de rayos $X$ Espectrómetro (EDX) Análisis de la Situación de la superficie metálica para la Distribución de Gas componentes del sistema. United States: ASTM International; 2012.

[12] ASTM B117-11. Standard Practice for Operating Salt Spray (Fog) Apparatus. United States: ASTM International; 2011.

[13] ASTM D3359-09e2. Standard Test Methods for Measuring Adhesion by Tape Test. United States: ASTM International; 2009.

[14] ASTM G5-94. Standard Reference Test Method for Making Potentiostatic and Potentiodynamic Anodic Polarization Measurements. United States: ASTM International; 2011.

[15] ASTM G59 - 97. Standard Test Method for Conducting Potentiodynamic Polarization Resistance Measurements. United States: ASTM International; 2014.

[16] ASTM G102 - 89. Standard Practice for Calculation of Corrosion Rates and Related Information from Electrochemical Measurements. United States: ASTM International; 2015.

[17] ASTM G1 - 03. Standard Practice for Preparing, Cleaning, and Evaluating Corrosion Test Specimens. United States: ASTM International; 2011. 